\title{
High Frequency Oscillatory Ventilation in Pediatric Practice: A Single Center Experience in Saudi Arabia
}

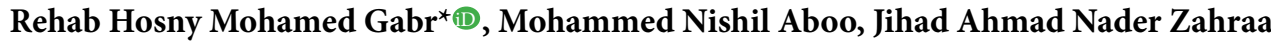

Pediatric Intensive Care Department, Children Specialized Hospital, King Fahad Medical City, PO Box 59046 Riyadh 11525, Saudi Arabia

\section{Abstract}

Publication History:

Background: High-frequency oscillatory ventilation (HFOV) is an advanced mode of mechanical Received: December 27, 2020 ventilation, however, its role has been a subject of debate. This study aimed to find patients who are best Accepted: January 23, 2021 treated with HFOV and the best selection of HFOV settings in the first $48 \mathrm{~h}$.

Published: January 252021

Methods: A retrospective study was conducted on 207 patients admitted to the pediatric intensive care unit ((PICU) in King Fahad Medical City and switched to HFOV between September 2010 and December 2017.

Results: The median age of our cohort was 12 (interquartile range [IRQ] 4-48) months, and 52.7\% were males. The most common cause of respiratory failure was acute respiratory distress syndrome (ARDS) with $100(48.3 \%)$ cases followed by pneumonia with 34 (16.4\%) cases. The majority (87.4\%) of participants had a chronic illness. Oxygenation failure was the most frequently reason for using HFOV (77.3\%). During first $48 \mathrm{~h}$, significant improvements in oxygenation and ventilation were noted. There was a significant association between $48 \mathrm{~h}$ mortality and lower $\mathrm{pH}$, sepsis, high vasopressor index, hypotension, hematological malignancies (odds ratio [OR] 8.73, 95\% confidence interval [CI] 1.58-48.06; $p=0.013$ ) and in a group that did not have diffuse alveolar or small airway diseases when compared with ARDS (OR 2.73, 95\% CI 1.13-6.58; $\mathrm{p}=0.030$ ).

Conclusion: Our study supports the value of HFOV in improving oxygenation and ventilation. Hemodynamic instability and sepsis are major contributors to worse short-term outcome in addition to the underlying diseases, mainly immune dysfunction and malignancies.

\section{Abbreviations}

HFOV: High frequency oscillatory ventilation, PICU: Pediatric Intensive Care Unit, ARDS: Acute Respiratory Distress Syndrome, FiO2: Fraction of inspired Oxygen, DAD: Diffuse alveolar disease, SAD: Small airway disease, MAP: Mean airway pressure, CMV: Conventional mechanical ventilation, ECMO: Extracorporeal membranous oxygenation, KFMC: King Fahad Medical City, OSI: Oxygen saturation index, VIS: Vasoactive-Inotropic Score, PIP: Peak inspiratory pressure, IRB: Institutional review board, CPAP: Continuous positive airway pressure, NIV: Non-invasive ventilation, iNO: Inhaled nitric oxide, NMB: Neuromuscular blocking agent, OI: Oxygenation index

\section{Introduction}

High frequency oscillatory ventilation (HFOV) is considered an advanced, unconventional, and presumably protective mode of mechanical ventilation used in selected cases of pediatric respiratory failure. By using HFOV, we recruit diseased lung and improve oxygenation, and to some degree ventilation, by constantly distension using high mean airway pressure (MAP) with tidal volume less than anatomic dead space. Several studies in medical literature have suggested more improvement, mainly in oxygenation and also in ventilation, in patients managed with HFOV when compared to conventional mechanical ventilation (CMV) [1-5]. Despite the wide use in all age groups and many success stories with this mode of HFOV, some newly published studies have described contradicting results. Recently, in two randomized, controlled, multicenter trials in adults, the use of HFOV was of questionable value and not associated with decreased mortality [6,7]. Even in the pediatric age group, some new studies have shown no clear benefit over continuous mandatory ventilation $(\mathrm{CMV})$ and even shows the tendency toward increased mortality and complications [8-10].

It is not clear thus far which factors contribute to the success or failure of the HFOV mode of ventilation. Several factors might contribute to the failure of HFOV: (1) underlying disease, (2) pathophysiology and disease nature, (3) degree and type of gas exchange impairment, (4) duration of CMV, and/or (5) maximum settings of previous conventional ventilation.
No standardized international practice guidelines are available for the proper indications for switching from conventional mode to HFOV, which cases will improve better on HFOV, and proper adjustment of the HFOV settings. Therefore, the objectives of this study were to identify which patient profiles could be best treated with HFOV in medical centers, especially with the lack of readily available extracorporeal membranous oxygenation (ECMO) rescue therapy, which ones will not improve or even worsen the condition, and the best possible way of selecting and adjusting HFOV settings in the first $48 \mathrm{~h}$.

\section{Materials and Methods}

A retrospective observational study was conducted in the pediatric intensive care unit (PICU) at King Fahad Medical City (KFMC), a tertiary care hospital located in Riyadh, the capital of Saudi Arabia, between September 2010 and December 2017. All patients under

*Corresponding Author: Dr. Rehab Hosny Mohamed Gabr, Pediatric Intensive Care Department, Children Specialized Hospital, King Fahad Medical City, PO Box 59046 Riyadh 11525, Saudi Arabia; E-mail: rehabgabr@yahoo.com

Citation: Gabr RHM, Aboo MN, Zahraa JAN (2021) High Frequency Oscillatory Ventilation in Pediatric Practice: A Single Center Experience in Saudi Arabia. Int J Pediatr Neonat Care 7: 171. doi: https://doi.org/10.15344/2455-2364/2021/171

Copyright: (c) $2021 \mathrm{Gabr}$ et al. This is an open-access article distributed under the terms of the Creative Commons Attribution License, which permits unrestricted use, distribution, and reproduction in any medium, provided the original author and source are credited. 
18 years of age with respiratory failure who were on CMV and then switched to HFOV were eligible to participate in this study. Patients whom were directly put on HFOV without previously being on CMV. Patients with incomplete electronic charts were excluded.

All included patients were ventilated using 3100-A HFOV machine (SensorMedics, Yorba Linda, California). Data were obtained from patients' medical records using an electronic chart viewer and collected on specially designed capture sheets by two pediatric intensivists working in the unit as the primary investigators. Collected data included patient's demographic data, hospital and PICU admission dates, background diseases if any, underlying cause of respiratory failure and its pathophysiology (diffuse alveolar disease [DAD], small airway disease [SAD], air leak, mixed pathology, and others), duration of CMV and HFOV, reasons for switching to HFOV, and survival at $48 \mathrm{~h}$. Ventilator settings, blood gases, oxygen saturation index (OSI), blood pressure (BP), and vasoactive-inotropic score (VIS) were all extracted just before connecting the patient to HFOV and 0, 2, 6, 12, 24 , and $48 \mathrm{~h}$ after connecting the patient to HFOV. In children, VIS is a reliable marker of cardiovascular support and provide additive value to existing pediatric acuity scores in this population [11].

Primary outcome was improvement versus worsening of OSI, $\mathrm{pH}$ $\mathrm{PCO}_{2}$, and vasoactive score. Secondary outcome was survival rate at $48 \mathrm{~h}$. The pathophysiology of the respiratory disease was determined based on the diagnoses as documented in the patient's medical record by the treating medical team. The diagnosis was confirmed by evaluating collected blood gases values, support provided on ventilator, and chest radiographic findings that were reviewed by the primary investigators and supported by the official radiology report. Oxygenation failure was defined as persistent desaturation below $90 \%$ while on $100 \%$ fraction of inspired oxygen $\left(\mathrm{FiO}_{2}\right)$ or when there is a need for at least $80 \% \mathrm{FiO}_{2}$ to maintain an $\mathrm{O}_{2}$ saturation above $90 \%$ or the need for more than $60 \% \mathrm{FiO}_{2}$ to maintain an $\mathrm{O}_{2}$ saturation of more than $84 \%$. Ventilation failure was defined as $\mathrm{pH}$ less than 7.30 with elevated $\mathrm{CO}_{2}$ compared to baseline. High settings were defined as peak inspiratory pressure (PIP) greater than $30 \mathrm{~cm} \mathrm{H}_{2} \mathrm{O}$. Hypotension was defined as systolic BP readings below the $5^{\text {th }}$ centile for age.

\section{Ethical considerations}

The study protocol was approved by KFMC institutional review board (IRB), and collected data were treated with strict confidentiality as per KFMC institutional regulations.

\section{Data Analysis}

Continuous data were presented as mean and standard deviation (SD) if normally distributed or median and interquartile range if the distribution was not normal. Categorical data were presented using frequencies and percentages. Continuous variables were compared using the independent-sample $t$-test or the Mann-Whitney $U$-test. Categorical variables were compared using the Fisher's exact or Pearson's chi-square test.

Differences were considered statistically significant when two-tailed $P$-values were less than 0.05 . Calculations were performed using SPSS Statistics, version 22.0 (SPSS Inc., Chicago, IL., USA).

\section{Results}

With a study period of over seven years, it was found that HFOV was used to support respiratory failure 241 times. Twenty-eight cases out of the 241 were excluded due to missing and important data in the medical records. Two patients were treated directly with HFOV without any conventional ventilation, and four patients were switched to HFOV from either the continuous positive airway pressure (CPAP) or non-invasive ventilation (NIV) modes; these cases were also excluded from the analysis. Two-hundred seven HFOV episodes were analyzed.

\section{Patient characteristics}

The median age of our cohort was 12 (interquartile range [IQR] 4-48) months with $52.7 \%$ male cases. The most common cause of respiratory failure that required HFOV rescue use was acute respiratory distress syndrome (ARDS) in 100 (48.3\%) cases followed by pneumonia in $34(16.4 \%)$ cases. Cases with small airway disease (SAD) represented only $4.9 \%$ of the studied cohort with cases of asthma and eight with bronchiolitis diagnoses. Cases with mixed pathophysiology of diffuse alveolar disease (DAD) with SAD accounted for $15 \%$ of the cases. A total of 32 out of the 207 cases had significant respiratory failure that required HFOV but did not fit into any of the commonly described indications for acute respiratory failure that usually benefit from HFOV. The majority $(87.4 \%)$ of the study patients had an underlying chronic illness, and the most frequently observed illnesses were malignancies (hematological and solid organ), neurological diseases, and immunodeficiency disorders $(15.5 \%, 13 \%$, and $12.6 \%$, respectively). Chronic lung disease was only encountered in 14 cases (6.8\%).

Oxygenation failure was the most frequently reported reason for shifting to HFOV followed by ventilation failure and then toxic high ventilatory settings, $(77.3 \%, 68.6 \%$, and $56 \%$ respectively) (Table 1 ). Most of our pre-HFOV cases were moderately to severely hypoxemic with a median OSI of 17.8 (IQR 14.3-22), acidotic with median $\mathrm{pH}$ pre-HFOV of 7.18 (IQR 7.06-7.28), and hypercarbic with median $\mathrm{PCO}_{2}$ pre-HFOV of 69 (54, 93 IQR). Inhaled nitric oxide (iNO) was tried before HFOV only in $24.6 \%$ of the cases, and a neuromuscular blocking agent was used before the transition in more than half of the cases (54.6\%). Hypotension while on conventional ventilation just before switching to HFOV was encountered in $19.3 \%$ of the cases and increased by almost two-fold during the first $48 \mathrm{~h}$ of commencing HFOV therapy (36.4\%).

\begin{tabular}{|l|l|l|}
\hline & Frequency & Percent \\
\hline Oxygenation failure & 160 & 77.3 \\
\hline Ventilation failure & 142 & 68.6 \\
\hline High settings & 116 & 56.0 \\
\hline Air leak & 1 & 0.5 \\
\hline Others & 4 & 1.9 \\
\hline Total & 207 & 100.0 \\
\hline
\end{tabular}

Table 1: Reason for shifting to high frequency oscillatory ventilation (HFOV).

\section{Outcome}

Over the first $48 \mathrm{~h}$ period after transition to rescue HFOV, a significant improvement in oxygenation parameters were observed in our cohort using this modality of ventilation associated with the need for gradual increment in mean airway pressure (Figure 1). The target of less than $60 \% \mathrm{FiO}_{2}$ was achieved in many of the cases within $24 \mathrm{~h}$, but the response was weaker on the second day. 

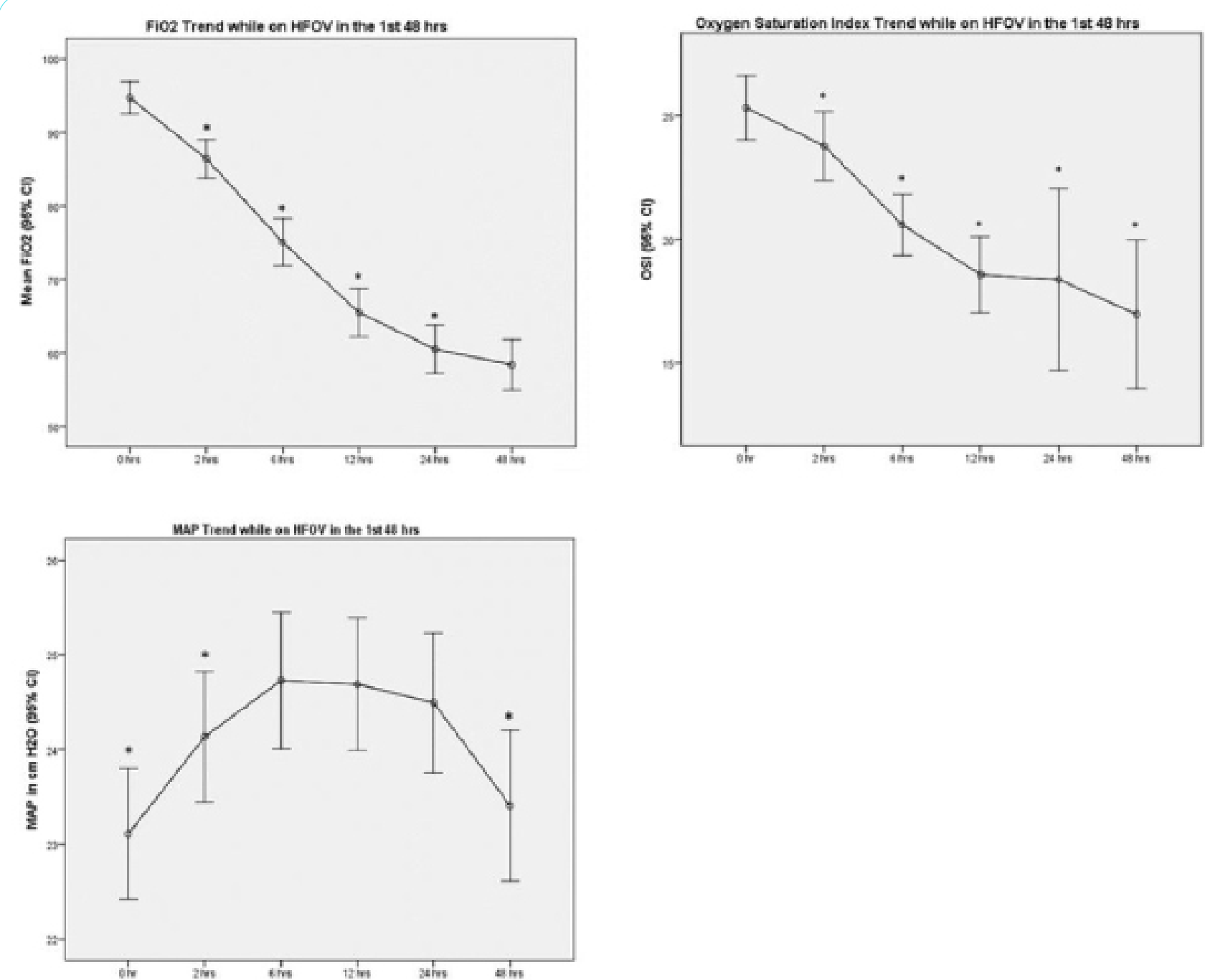

Figure 1: Mean changes and 95\% CI in Fraction of inspired $\mathrm{O}_{2}\left(\mathrm{FiO}_{2}\right)$, Oxygen Saturation and Mean Airway Pressure (MAP) for $48 \mathrm{~h}$ after application of high frequency oscillatory ventilation.

The parameters at $0 \mathrm{~h}$ were recorded immediately after starting high frequency oscillatory ventilation (HFOV). Values of each parameter were compared with preceding parameter using Wilcoxon signed ranks test. ${ }^{\star} \mathrm{p}<0.05$

The same improving trend in ventilation and acid base status was observed but was faster with the target being achieved within 6 to 12 $\mathrm{h}$ (Figure 2). The change was associated with an increment of HFOV amplitude but more significant titration of frequency of oscillation was observed in our group $(p<0.05)$.

\section{Short term (48 h) mortality risk}

Table 2 shows patients' demographics and variables related to illness severity, underlying conditions, pathophysiology, and its possible impact on short-term survival. The overall $48 \mathrm{~h}$ survival of HFOV rescue therapy was achieved in $82.2 \%$ of the cases. No significant differences between patients who survived 48 hand the non-survivors in terms of age, weight, height, gender, presence of underlying diagnosis, prior to the use of $\mathrm{iNO}, \mathrm{O}_{2} \mathrm{OSI}$, and pre-HFOV $P-\mathrm{CO}_{2}$ were noted $(p>0.05)$.

The lower chance of short-term survival was associated with shorter duration of $\mathrm{CMV}$, lower $\mathrm{pH}$, not using neuromuscular blocking agents, presence of sepsis, high vasopressor index, and hypotensive status just before switching to the rescue therapy or within $48 \mathrm{~h}$ of initiation of HFOV therapy $(p<0.05)$.

Table 3 shows the 48-hmortality based on different groups of underlying diseases. The only significant association in our cohort was found with patients with hematological malignancies (OR 8.73, 95\% CI 1.58-48.06; $p=0.013$ ). Patients with underlying cardiac disease or immunodeficiencies had higher mortality rates compared to normal (control) patients, but this difference did not reach statistical significance. The short term 48-h survival in patients who were rescued for ARDS using HFOV was $82 \%$. When using ARDS as a reference group, the only statistically significant difference in mortality was seen in the poorly identified group that did not fit into either the DAD or SAD categories (Table 4).

The $48 \mathrm{~h}$ survival rates in cases of oxygenation and/or ventilation failures and high settings were $83.2 \%, 79.7 \%$, and $83.2 \%$ respectively ( $p=0.016)$. CMV settings showed no significant difference between surviving versus non-surviving groups (Table 5). 
Citation: Gabr RHM, Aboo MN, Zahraa JAN (2021) High Frequency Oscillatory Ventilation in Pediatric Practice: A Single Center Experience in Saudi Arabia. Int J Pediatr Neonat Care 7: 171. doi: https://doi.org/10.15344/2455-2364/2021/171
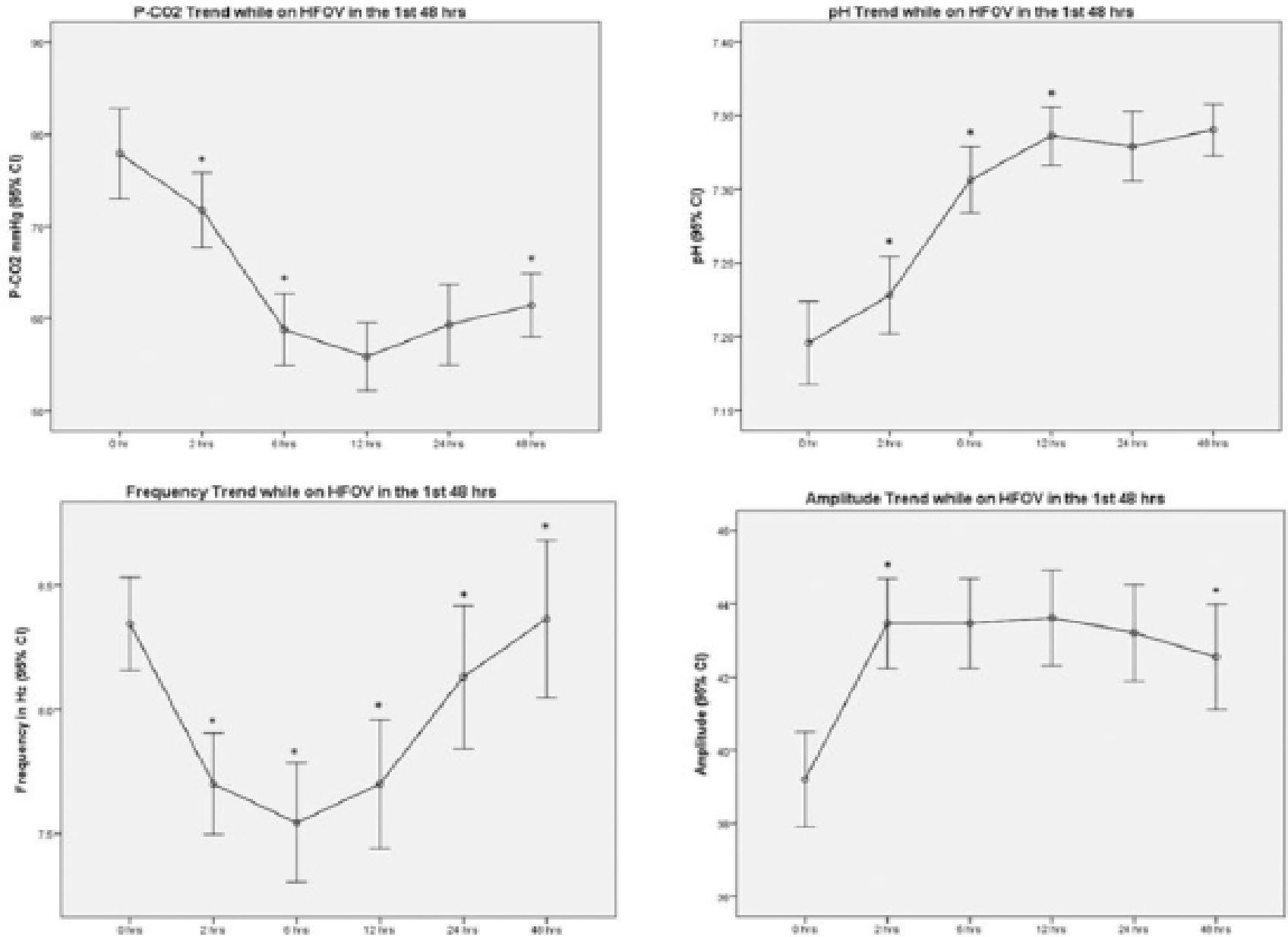

Figure 2: Mean changes and 95\% confidence interval (CI) in partial pressure of $\mathrm{CO}_{2}\left(\mathrm{PCO}_{2}\right), \mathrm{pH}, \mathrm{HFOV}$ frequency, and amplitude for $48 \mathrm{~h}$ after application of HFOV ventilation.

The parameters at 0 hour are the parameters recorded immediately after starting HFOV. Values of each parameter were compared with preceding parameter using the Wilcoxon signed rank test. ${ }^{\star} P<0.05$

\begin{tabular}{|c|c|c|c|c|}
\hline \multirow[b]{2}{*}{ Variables } & & \multicolumn{2}{|c|}{48 h Survival } & \multirow[b]{2}{*}{$P$-values } \\
\hline & & Survivors $n=170$ & Non-survivors $n=37$ & \\
\hline Age (month) & $12(4,48)$ & $12(4,48)$ & $11(3,43)$ & 0.85 \\
\hline Weight (kg) & $7.4(4.1,12.2)$ & $7.7(4.2,12)$ & $6(3.4,13)$ & 0.53 \\
\hline Height $(\mathrm{cm})$ & $65(53,92)$ & $67(54,91.5)$ & $60(51.8,103)$ & 0.80 \\
\hline Male & $109(52.7 \%)$ & $86(50.6 \%)$ & $23(62.2 \%)$ & 0.21 \\
\hline OSI Pre-HFOV & $17.8(14.3,22)$ & $18(14.4,22.1)$ & $17.5(6,33.3)$ & 0.63 \\
\hline CMV days pre HFOV & $1.25(0.5,6)$ & $2(1,6)$ & $1(0.3,5.5)$ & 0.046 \\
\hline pH pre-HFOV & $7.18(7.06,7.28)$ & $7.21(7.10,7.31)$ & $6.99(6.85,7.20)$ & $<0.001$ \\
\hline $\mathrm{PCO}_{2}$ pre-HFOV & $69(54,93)$ & $69(54,95.3)$ & $68(50.1,91)$ & 0.77 \\
\hline Vasopressor index pre-HFOV & $(5,50)$ & $0(0,21.3)$ & $70(10,190)$ & $<0.001$ \\
\hline Sepsis (associated diagnosis) & $85(41 \%)$ & $59(34.7 \%)$ & $26(70.3 \%)$ & $<0.001$ \\
\hline Underlying chronic illness & $181(87.4 \%)$ & $146(85.9 \%)$ & $35(94.6 \%)$ & 0.18 \\
\hline iNO use & $51(24.6 \%)$ & $46(27.1 \%)$ & $5(13.5 \%)$ & 0.095 \\
\hline NMB agent use & $113(54.6 \%)$ & $104(61.2 \%)$ & $9(24.3 \%)$ & $<0.001$ \\
\hline Hypotension Pre-HFOV & $40(19.3 \%)$ & $27(15.9 \%)$ & $13(35.1 \%)$ & 0.011 \\
\hline Hypotension anytime during the first $48 \mathrm{hrs}$ of HFOV & $75(36.4 \%)$ & $48(28.2 \%)$ & $27(75.0 \%)$ & $<0.001$ \\
\hline
\end{tabular}

Table 2: Baseline demographics and patient characteristics with $48 \mathrm{~h}$ survival.

Significant variables presented in bold.

OSI: oxygen saturation index; CMV: conventional mechanical ventilation, iNO: inhaled nitric oxide; NMB: neuromuscular blocking agent. Data are presented as either a number and percentage or median and interquartile range (IQR). 
Citation: Gabr RHM, Aboo MN, Zahraa JAN (2021) High Frequency Oscillatory Ventilation in Pediatric Practice: A Single Center Experience in Saudi Arabia. Int J Pediatr Neonat Care 7: 171. doi: https://doi.org/10.15344/2455-2364/2021/171

Page 5 of 7

\begin{tabular}{|l|l|l|l|l|l|}
\hline Underlying Disease & Total $n(n \%)$ & Survived $n(n \%)$ & Died $n(n \%)$ & OR $(95 \%$ CI $)$ & $P$-value \\
\hline No chronic illness & $26(12.6)$ & $24(92.3)$ & $2(7.7)$ & 1 & \\
\hline Chronic illness & $181(87.4)$ & $145(80.6)$ & $35(19.4)$ & $2.90(0.65-12.84)$ & 0.180 \\
\hline Underlying Disease & $19(9.2)$ & $11(57.9)$ & $8(42.1)$ & \multicolumn{3}{|l|}{$\mathbf{8 . 7 3 ( 1 . 5 8 - 4 8 . 0 6 )}$} & $\mathbf{0 . 0 1 3}$ \\
\hline Hematologic & $13(6.3)$ & $12(92.3)$ & $1(7.7)$ & $1.00(0.08-12.16)$ & 1.000 \\
\hline Solid organ malignancy & $26(12.6)$ & $20(76.9)$ & $6(23.1)$ & $3.60(0.65-19.84)$ & 0.141 \\
\hline Immunodeficiency & $15(7.2)$ & $13(86.7)$ & $2(13.3)$ & $1.85(0.23-14.67)$ & 0.562 \\
\hline Metabolic & $14(6.8)$ & $12(85.7)$ & $2(14.3)$ & $2.00(0.25-15.99)$ & 0.513 \\
\hline Chronic lung disease & $10(4.8)$ & $9(90.0)$ & $1(10.0)$ & $1.33(0.11-16.57)$ & 0.823 \\
\hline Chronic leaver disease & $6(2.9)$ & $5(83.3)$ & $1(16.7)$ & $2.40(0.18-31.88)$ & 0.507 \\
\hline Chronic kidney disease & $27(13.0)$ & $24(88.9)$ & $3(11.1)$ & $1.50(0.23-9.80)$ & 0.672 \\
\hline Chronic neurologic disease & $8(3.9)$ & $7(87.5)$ & $1(12.5)$ & $1.71(0.13-21.82)$ & 0.678 \\
\hline Syndromic & $5(2.4)$ & $5(100.0)$ & $0(.0)$ & $1.20(0.05-30.81)$ & 0.999 \\
\hline Skeletal abnormality & $16(7.7)$ & $11(68.8)$ & $5(31.3)$ & $5.45(0.91-32.62)$ & 0.063 \\
\hline Cardiac & $22(10.6)$ & $17(77.3)$ & $5(22.7)$ & $3.53(0.61-20.38)$ & 0.159 \\
\hline Others & & & & \\
\hline
\end{tabular}

Table 3: 48 h mortality per presence of underlying disease.

Significant variable presented in bold.

\begin{tabular}{|l|l|l|l|l|l|}
\hline Cause of respiratory failure & Total $n(n \%)$ & Survived $n(n \%)$ & Died $n(n \%)$ & OR of mortality $(95 \% \mathrm{CI})$ & $P$-value \\
\hline ARDS & $100(48.3)$ & $82(82.0)$ & $18(18.0)$ & 1 & \\
\hline Bronchiolitis & $8(3.9)$ & $8(100.0)$ & $0(0.0)$ & $0.28(0.02-5.19)$ & 0.347 \\
\hline Asthma & $2(1.0)$ & $2(100.0)$ & $0(0.0)$ & $1.14(0.05-26.33)$ & 1.000 \\
\hline Mixed Disease & $31(15.0)$ & $29(93.5)$ & $2(6.5)$ & $0.31(0.07-1.44)$ & 0.157 \\
\hline Pneumonia & $34(16.4)$ & $29(85.3)$ & $5(14.7)$ & $0.79(0.27-2.31)$ & 0.795 \\
\hline Other & $32(15.5)$ & $20(62.5)$ & $12(37.5)$ & $\mathbf{2 . 7 3 ( 1 . 1 3 - 6 . 5 8 )}$ & $\mathbf{0 . 0 3 0}$ \\
\hline
\end{tabular}

Table 4: Short-term $48 \mathrm{~h}$ survival per cause of respiratory failure.

Significant variable presented in bold.

\begin{tabular}{|l|l|l|l|}
\hline & \multicolumn{2}{|c|}{ 48-hour survival } & \\
\hline Variables & Survived & Died & $P$-value \\
\hline Fraction of inspired $\mathrm{O}_{2}\left(\mathrm{FiO}_{2}\right)$ & $88.44(17.70)$ & $87.97(20.05)$ & 0.887 \\
\hline Positive End Expiratory Pressure (PEEP) & $9.43(2.45)$ & $9.35(2.35)$ & 0.855 \\
\hline Peak Inspiratory Pressure (PIP) & $31.60(4.69)$ & $31.86(5.53)$ & 0.768 \\
\hline Plateau Pressure (P-Plat) & $29.29(3.64)$ & $30.92(4.10)$ & 0.192 \\
\hline Mean Airway Pressure (MAP) & $17.84(3.79)$ & $18.06(3.69)$ & 0.761 \\
\hline Respiratory Rate (RR) & $36.57(9.81)$ & $37.70(8.24)$ & 0.513 \\
\hline Inspiratory Time (Ti) & $0.59(0.18)$ & $0.59(0.16)$ & 0.972 \\
\hline Expiratory Time (Te) & $1.15(0.45)$ & $1.11(0.44)$ & 0.644 \\
\hline Tidal Volume $(\mathrm{Vt}) \mathrm{ml} / \mathrm{kg}$ & $8.23(3.54)$ & $8.11(3.02)$ & 0.857 \\
\hline Oxygen Saturation $\left(\mathrm{O}_{2}\right.$-sat) & $87.29(11.81)$ & $88.79(11.06)$ & 0.503 \\
\hline
\end{tabular}

Table 5: Pre-HFOV mechanical ventilation setting and its association with 48 -h survival.

$\mathrm{P}<0.05$ is considered significant.

\section{Discussion}

As the debate about the value of HFOV in pediatric respiratory failure refractory to conventional means continues, the greater the need to share different experiences from different areas of the world to try to add to our understanding of this unresolved issue. We were able to demonstrate the clear value of HFOV rescue therapy in improving both oxygenation and ventilation as has been described by others from different areas of the world in our study population [1-5]. The majority of our patients had DAD as a pathophysiology, which explained the failure of both oxygenation and ventilation. HFOV was also used in our group for small numbers of SAD, which was very successful after being refractory to conventional ventilation strategies. Patients' demographics and CMV settings did not have significant impact on short term survival in our cohort. 
In our patients, a remarkable association of number of days on CMV with the patient outcome was noted as patients who died within $48 \mathrm{~h}$ had in average a shorter duration of CMV ( $p=0.046)$. The other factors that had an impact on 48-hsurvival were the last $\mathrm{pH}$ reading, presence of sepsis in the diagnoses, and hypotension just prior or during the $48 \mathrm{~h}$ of HFOV use $(p<0.01)$. The use of pre-HFOV neuromuscular blocking agents was observed more with the survivors $(p<0.001)$ and also with the trial of inhaled nitric oxide, but it did not reach statistical significance in the latter $(p=0.095)$.

Our results showed a higher mortality rate in patients with underlying hematological malignancies and to a lesser degree, patients with cardiac problems and immunodeficiencies. Based on the cause of respiratory failure and after comparing different categories with ARDS group, we were able to show an association of worse short-term outcome in the group that had respiratory failure but did not fit clinically or radiologically the findings in DAD or SAD, the commonly described indications for HFOV (OR of mortality 2.73, 1.13-6.58); $\mathrm{p}=0.030)$.

In a recent publication, Retting et al. described an updated multicenter experience using HFOV in pediatric acute lung injury [12]. They emphasized in their publication, based on results from different centers from North America and Europe, the importance of underlying conditions and severity of hypoxemia, as measured by oxygenation index (OI) for predicting survival. For their patients, an immunocompromised state followed by cyanotic heart disease and then chronic lung disease were the cases that had the highest probability of death. Similarly, in our study we observed almost same pattern but to a lesser degree even though we are only addressing short-term mortality. Moreover, our study showed a higher mortality in patient with hematological malignancies. The higher mortality when using HFOV for refractory hypoxemia associated with malignancies and in post-stem cell transplant cases has been described by more than one recent publication $[13,14]$. These cases deserve the highest degree of vigilance and need additional well-designed trials to account for all possible confounding variables. We could not duplicate the findings of worse associated outcomes as reported by Retting et al. or the severity of hypoxemia as measured by OI. An interesting finding in our cohort was the association of short-term outcome with the hemodynamic variables. The significant association of lower $\mathrm{pH}$ value (not related to $\mathrm{pCO}_{2}$ ), hypotension before and during the HFOV trial, higher vasopressor scores, and presence of sepsis as secondary diagnosis with mortality is something to be further examined. The use of HFOV and its potential associated impact on cardiac function and hemodynamic instability especially in dehydrated patient is not new to medical literature $[15,16]$. This fact deserves special consideration when choosing this therapy modality after considering evolving evidence and the "hot" discussion questioning the value of HFOV when compared with more proper applications of CMV [8-10]. We believe in the value of using HFOV in our hospital, especially with the lack of ECMO support for the time being, but the burning questions will always be "Who will benefit the most?" and "How do we apply this mode of ventilation properly?".

One more observation we noticed in our cohort after looking at the adjustment of the ventilator settings with high $\mathrm{CO}_{2}$. It was obvious from the data that we have been turning the frequency down more than increasing the amplitude. This practice is probably suboptimal and different from what is currently being recommended and practiced by other intensivists utilizing higher $\mathrm{Hz}$ and higher delta $\mathrm{P}$ strategies to protect the lung from high tidal volume injury.
This study is the first to address these points in the kingdom of Saudi Arabia, and hopefully it will help create unified guidelines for HFOV use and standardize PICU practices in this regard.

The retrospective design and data collection difficulties due to suboptimal archiving and the transition period to electronic health record were the major limitations of our study. Blood gases were mainly obtained from capillaries; thus, oxygenation index could not be used, and the surrogate OSI was utilized in our cases as it is proved by recent publication to be an acceptable alternative (as discussed in recent publications) to measure the severity of hypoxemia and is being used as an alternative measurement in the most recent definition of pediatric ARDS [17]. Another limitation of our study is the lack of detailed data on the 32 out of the 207 cases that had significant respiratory failure and were placed on HFOV. These cases did not fit into the DAD or SAD categories, which are the most frequently studied indications of acute respiratory failure that are managed by HFOV. These cases are worthy of analyzing as they presented a higher mortality risk, but this was beyond the scope of our current study.

Despite these limitations, we believe our study adds some insight about some of the factors that can be associated with short term improvement and mortality. These factors need to be taken into consideration when we choose to apply the HFOV modality as rescue therapy. Choosing the right patient, the right time, and the right technique will surely help us provide more convincing evidence of the value of this mode of ventilation. Having a good randomized clinical trial addressing these variables will be of great help to the pediatric physicians working in the intensive care to guide them to make the right choices for their patients.

\section{Conclusion}

Our study supports the great value of HFOV in improving oxygenation and ventilation when the conventional mode fails to achieve its goals. The underlying disease seems to have a significant impact on the outcome. In our population, we were able to confirm some of the already described known risk factors for mortality and unable to replicate others. Hemodynamic instability and sepsis are major contributors to worse outcomes and should be tackled vigilantly. We still need to learn more about the proper selection of patients, time of switching to HFOV, and the proper safe technique to apply HFOV in pediatric respiratory failure.

\section{Competing Interests}

The authors declare that they have no competing interests.

\section{Acknowledgements}

We thank Ms. Michele Mella our department secretary for her help in entering the patient data collected into the electronic format. We thank Mr. Tariq Wani for his assistance performing the statistical analysis of our data.

\section{Funding}

The research was funded by King Fahad Medical City intramural research fund (Study number 017-056) to encourage research culture in the institution. 
Citation: Gabr RHM, Aboo MN, Zahraa JAN (2021) High Frequency Oscillatory Ventilation in Pediatric Practice: A Single Center Experience in Saudi Arabia. Int J Pediatr Neonat Care 7: 171. doi: https://doi.org/10.15344/2455-2364/2021/171

Page 7 of 7

There was no role for the funding body in the design of the study and collection, analysis, and interpretation of data and in writing the manuscript.

\section{Authors' Contributions}

Rehab Gabr did the research proposal, collected the data, refined it, assisted in data analysis and wrote the manuscript draft.

Mohammed Aboo helped in data collection, refining it, assisted in writing the manuscript.

Jihad Zahraa helped refining the proposal, refining the data, data analysis and in finalizing the manuscript. All authors read and approved the final manuscript.

All had given the permission for submission.

\section{References}

1. Yu-Xiong Guo YX, Wang ZN, Li YT, Pan L, Yang LF, et al. (2016) High frequency oscillatory ventilation is an effective treatment for severe pediatric acute respiratory distress syndrome with refractory hypoxemia. Ther Clin Risk Manag 12: 1563-1571.

2. Ben Jaballah N, Khaldi A, Mnif K, Bouziri A, Belhadj S, et al. (2006) Highfrequency oscillatory ventilation in pediatric patients with acute respiratory failure. Pediatr Crit Care Med 7: 362-367.

3. Moniz M, Silvestre C, Nunes P, Abadesso C, Matias E, et al. (2013) High frequency oscillatory ventilation in children: a 10-year experience. J Pediatr 89: 48-55.

4. Fioretto JR, Klefens SO, Pires RF, Kurokawa CS, Carpi MF, et al. (2017) Comparison between conventional protective mechanical ventilation and high-frequency oscillatory ventilation associated with the prone position. Rev Bras Ter Intensiva 29: 427-435.

5. Slee-Wijffels FY, van der Vaart KR, Twisk JW, Markhorst DG, Plötz FB, et al. (2005) High-frequency oscillatory ventilation in children: a single-center experience of 53 cases. Critical Care 9: R274-R279.

6. Young D, Lamb SE, Shah S, MacKenzie I, Tunnicliffe W, et al. (2013) High Frequency Oscillation for Acute Respiratory Distress Syndrome. N Engl J Med 368: 806-813.

7. Ferguson ND, Cook DJ, Guyatt GH, Mehta S, Hand L, et al. (2013) Highfrequency oscillation in early acute respiratory distress syndrome. $\mathrm{N}$ Engl J Med 368: 795-805.

8. Gupta P, Green JW, Tang X, Gall CM, Gossett JM, et al. (2014) Comparison of high-frequency oscillatory ventilation and conventional mechanical ventilation in pediatric respiratory failure. JAMA Pediatr 168: 243-249.

9. Bateman ST, Borasino S, Asaro L, Cheifetz I, Diane S, et al. (2016) Early highfrequency oscillatory ventilation in pediatric acute respiratory failure. a propensity score analysis. Am J Respir Crit Care Med 193: 495-503.

10. Sud S, Sud M, Friedrich JO, Wunsch H, Meade MO, et al. (2016) High frequency oscillatory ventilation versus conventional ventilation for acute respiratory distress syndrome. Cochrane Database Syst Rev 4: CD004085.

11. McIntosh AM, Tong S, Deakyne SJ, Davidson JA, Scott HF, et al. (2017) Validation of the Vasoactive-Inotropic Score in Pediatric Sepsis. Pediatr Crit Care Med 18: 750-757.

12. Rettig JS, Smallwood CD, Walsh BK, Rimensberger PC, Bachman TE, et al (2015) High-frequency oscillatory ventilation in pediatric acute lung injury: a multicenter international experience. Crit Care Med 43: 2660-2667.

13. Rowan CM, Smith LS, Loomis A, McArthur J, Gertz SJ, et al. (2017) Pediatric acute respiratory distress syndrome in pediatric allogeneic hematopoietic stem cell transplants: a multicenter study. Pediatr Crit Care Med 18: 304309.

14. Stewart CA, Yehya N, Fei L, Chima RS (2018) High frequency oscillatory ventilation in a cohort of children with respiratory failure. Pediatr Pulmonol 53: 816-823.

15. David M, von Bardeleben RS, Weiler N, Karmordt J, Herweling A, et al. (2004) Cardiac function and haemodynamics during transition to high-frequency oscillatory ventilation. Eur J Anaesthesiol 21: 944-952.

Int J Pediatr Neonat Care

ISSN: 2455-2364
16. Smailys A, Mitchell JR, Doig CJ, Tyberg JV, Belenkie I, et al. (2014) High-frequency oscillatory ventilation versus conventional ventilation: hemodynamic effects on lung and heart. Physiol Rep 2: e00259.

17. Jouvet $\mathrm{P}$, Thomas N, Wilson D, Erickson S, Khemani R, et al. (2015) Pediatric acute respiratory distress syndrome: consensus recommendations from the Pediatric Acute Lung Injury Consensus Conference. Pediatr Crit Care Med 16: 428-439. 